\title{
Chitin nanofibril-based flame retardant for paper application
}

Tongling Zhang, ${ }^{\dagger, \dagger}$ Shigenori Kuga,${ }^{\dagger}$ Min Wu, ${ }^{*}, \dagger, \dagger$ Yong Huang ${ }^{*}, \dagger$

$\dagger$ Technical Institute of Physics and Chemistry, Chinese Academy of Sciences, Beijing 100190, China

$\$$ Center of Materials Science and Optoelectronics Engineering, University of Chinese Academy of Sciences, Beijing, 100039, China

*Min Wu. E-mail: wumin@mail.ipc.ac.cn. Tel: +86-10-82543500. Address: No. 29, Zhongguancun East Road, Haidian District, Beijing, 100190.

*Yong Huang. E-mail: yhuang@mail.ipc.ac.cn. Tel: +86-10-82543478. Address: No.

29, Zhongguancun East Road, Haidian District, Beijing, 100190. 
Number of Pages: 3

Number of Figures: 3
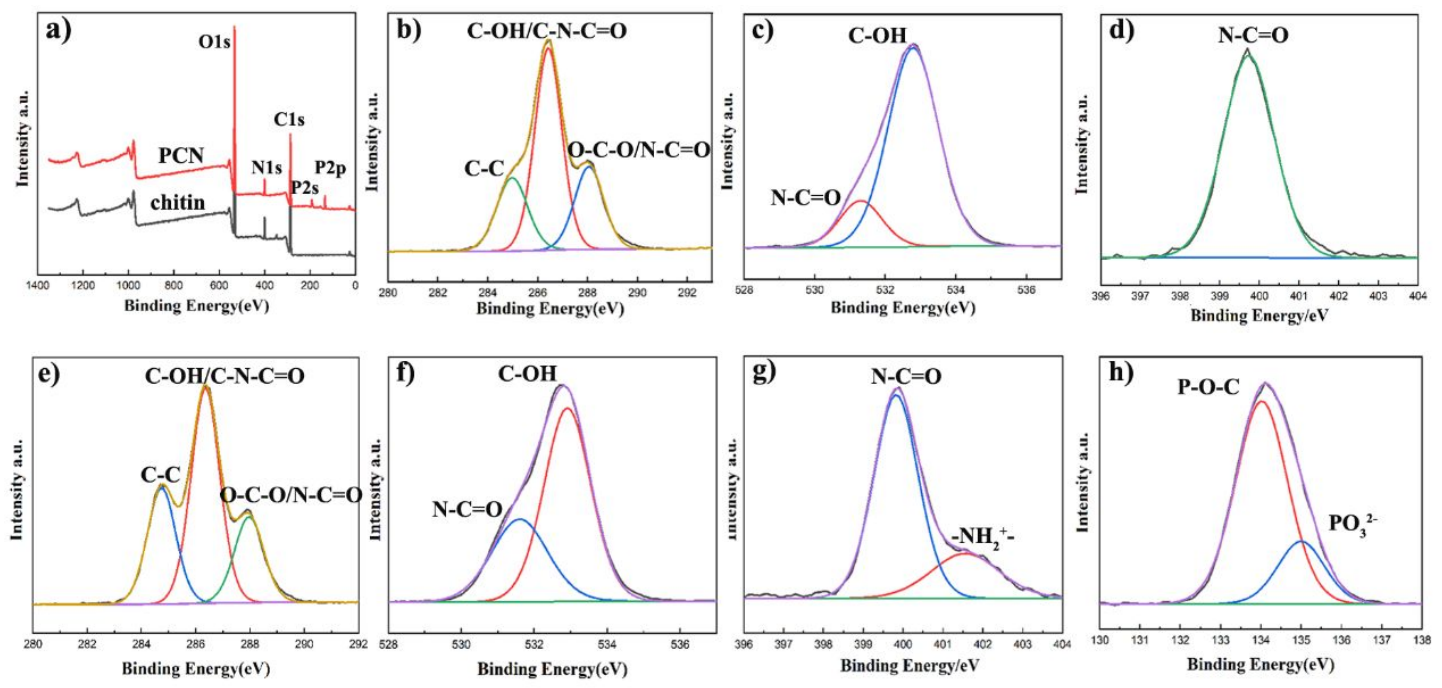

Figure S1. XPS spectrum of the chitin and chitin nanofibril-based flame retardant: a) the full spectrum of the chitin and chitin nanofibril-based flame retardant, b-d) the C1s, $\mathrm{O} 1 \mathrm{~s}$ and N1s spectrum of chitin, respectively; e-h) the $\mathrm{C} 1 \mathrm{~s}, \mathrm{O} 1 \mathrm{~s}, \mathrm{~N} 1 \mathrm{~s}$ and $\mathrm{P} 2 \mathrm{p}$ spectrum of chitin nanofibril-based flame retardant. 


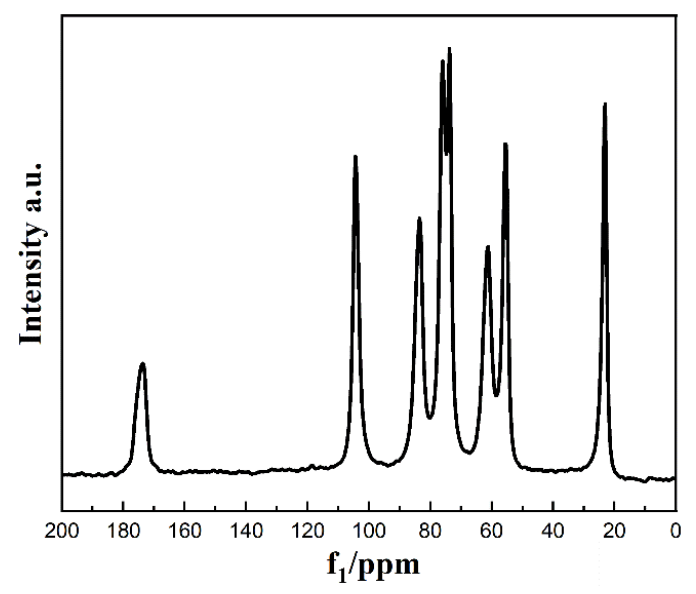

Figure S2. ${ }^{13} \mathrm{C} \mathrm{CP} / \mathrm{MAS}$ NMR spectrum of chitin.

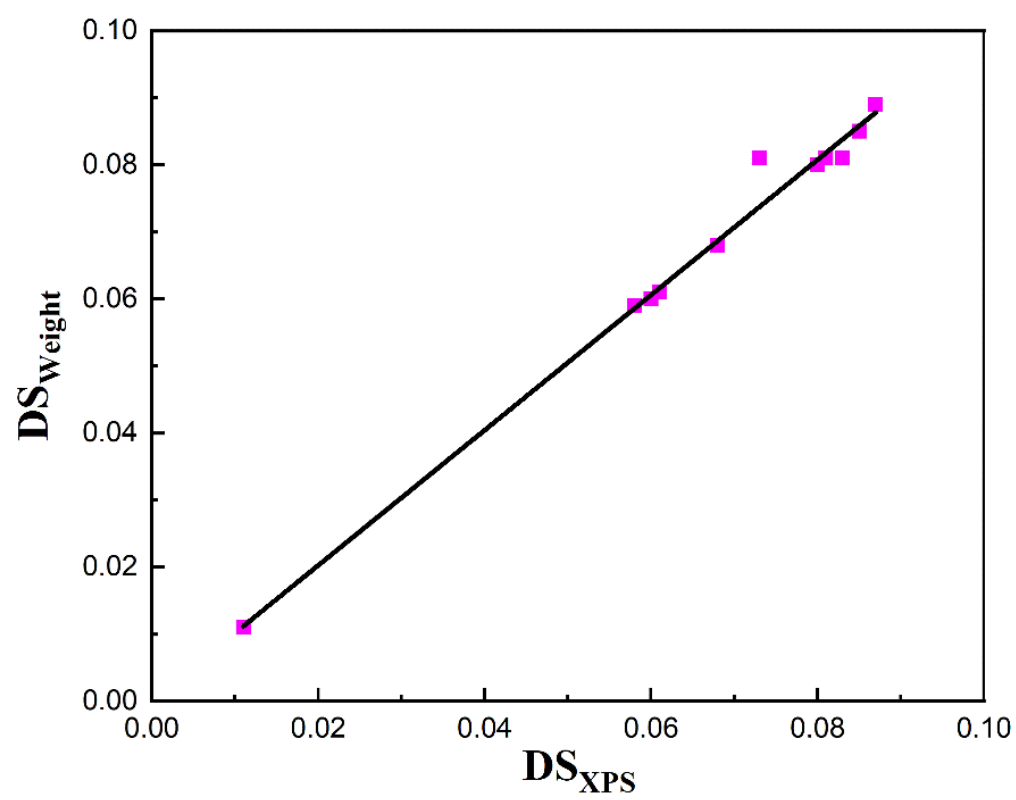

Figure S3. The relationship between $\mathrm{DS}_{\mathrm{Weight}}$ and $\mathrm{DS}_{\mathrm{XPS}}$. 